\title{
Significance of Tumor-Infiltrating Immune Cells in Spontaneous Canine Mammary Gland Tumor: 140 Cases
}

\author{
Kohei SAEKI ${ }^{1)}$, Yoshifumi ENDO ${ }^{1)}$, Kazuyuki UCHIDA ${ }^{2)}$, Ryohei NISHIMURA ${ }^{3)}$, Nobuo SASAKI ${ }^{1)}$ and \\ Takayuki NAKAGAWA ${ }^{1) *}$ \\ ${ }^{1)}$ Laboratory of Veterinary Surgery, Graduate School of Agricultural and Life Sciences, The University of Tokyo, Bunkyo-ku, Tokyo 113- \\ 8657, Japan \\ ${ }^{2)}$ Laboratory of Veterinary Pathology, Graduate School of Agricultural and Life Sciences, The University of Tokyo, Bunkyo-ku, Tokyo 113 \\ 8657, Japan \\ ${ }^{3)}$ Laboratory of Emergency Medicine, Graduate School of Agricultural and Life Sciences, The University of Tokyo, Bunkyo-ku, Tokyo 113 \\ 8657, Japan
}

(Received 16 March 2011/Accepted 12 September 2011/Published online in J-STAGE 22 September 2011)

ABSTRACT. The numbers of tumor infiltrating T lymphocytes, B lymphocytes and antigen presenting cells were evaluated in an immunohistochemical manner in 140 canine spontaneous mammary gland tumor (MGT) tissues. As a result, we found a statistically significant increase in the number of intratumoral T lymphocytes $(23.2 \pm 23.8)$ in the malignant MGT group $(\mathrm{n}=51)$ compared with the benign MGT group $(14.0 \pm 16.0, \mathrm{n}=89 ; P<0.05)$. Moreover, the high $\mathrm{T}$ lymphocyte infiltration in the malignant group correlated with poor prognosis in multivariate analysis $(P<0.05)$. This study indicated the relationship between increased infiltrating T lymphocytes and canine MGT malignancy. KEY WORDS: canine, CD3, mammary gland tumor, microenvironment, tumor-infiltrating leukocyte.

doi: 10.1292/jvms.11-0118; J. Vet. Med. Sci. 74(2): 227-230, 2012

Tumor tissues contain abundant types of cells such as innate immune cells (including macrophages, neutrophils, mast cells, myeloid-derived suppressor cells and natural killer cells) and adaptive immune cells ( $\mathrm{T}$ and B lymphocytes) in addition to tumor cells and surrounding stromal cells (fibroblasts, endothelial cells, pericytes and mesenchymal cells) $[4,10,15]$. Development of a tumor is considered to occur as a consequence of genetic aberrations as well as interplays between the cells mentioned above $[6,15]$. In this context, the host immune system is reported to play opposing and paradoxical roles $[6,10,33]$. There may be dynamic crosstalk between infiltrating leukocytes in tumor tissues and tumor cells, and whether they finally work in favor of or against tumors might be determined by their surrounding environment [10].

Canine mammary gland tumors (CMGTs) are the most common tumors in intact female dogs. CMGT is reported to account for approximately $50 \%$ of all tumors that naturally occur in female dogs $[7,20,25]$. Spontaneous CMGTs show many aspects, including epidemiological, clinical, biological and genetic characteristics, similar to those in human breast cancers. Therefore, several studies have investigated CMGTs as comparative models of human counterparts $[18,19,32]$.

With flow cytometric analysis, the recent research revealed that overall lymphocytic infiltration intensity cor-

\footnotetext{
* Correspondence to: Nakagawa, T., Laboratory of Veterinary Surgery, Graduate School of Agricultural and Life Sciences, The University of Tokyo, 1-1-1 Yayoi, Bunkyo-ku, Tokyo 1138657, Japan.

e-mail: anakaga@mail.ecc.u-tokyo.ac.jp
}

(C)2012 The Japanese Society of Veterinary Science related with poor prognosis in CMGT, and an increase in the $\mathrm{CD}^{+} / \mathrm{CD}^{+}$ratio was observed in patients with metastatic disease. Other reports dealing with small animal tumors investigated the relationship between leukocyte infiltration and cancer malignancy, such as mast cells in melanoma and the number of $\mathrm{CD}^{+}$and regulatory $\mathrm{T}$ cells in peripheral blood in metastatic diseases $[13,26]$. However, the knowledge about inflammation and immunity in cancer tissues is still limited in small animal oncology.

The purpose of this study was to improve the understanding of the interaction of immune cells with a tumor over the course of tumor progression in CMGT. Therefore, we conducted immunohistochemical analysis of infiltrating antigen-presenting cells (APCs) and adaptive immune cells ( $\mathrm{T}$ lymphocytes and B lymphocytes) in CMGT tissues of spontaneous patients and evaluated their relationships with clinicopathological features and overall survival.

We collected CMGT tissues from dogs that underwent surgical resection at the Veterinary Medical Center, the University of Tokyo, between April 1996 and December 2008. Tumor tissues were histologically classified by one pathologist according to the International Histological Classification of Tumors of Domestic Animals of the World Health Organization [24]. Benign tumor tissues from animals that had a history of malignant mammary tumor before or from animals that had developed malignant neoplasia simultaneously at surgery in other mammary glands were excluded in this study because of their potential malignancy. Eventually, a total of 140 CMGT tissues from 135 dogs were used in this study. Four dogs developed adenocarcinoma several years after the first resection of a benign mixed tumor. Two simple adenocarcinoma samples were obtained from a 
patient that underwent surgeries at two different time points. The other 130 samples were collected from 130 different patients. The samples were histologically divided into malignant $(n=51)$ and benign tumor groups $(n=89)$.

Medical records of patients were also reviewed, and clinical data were collected. Regional lymph node involvement was confirmed by biopsy and a subsequent histopathological examination, and lung metastasis was confirmed by thoracic radiography. In some cases, in which distant metastasis, excluding the lung, was suspected, additional examinations such as abdominal radiography and ultrasonography were performed. The WHO clinical staging was used for classification of the patients.

Immune cell infiltrations into tumor tissues were analyzed by immunohistochemistry for serial sections. The primary antibodies used in this analysis and their dilutions were as follows: anti-human CD20 rabbit polyclonal antibody at 1:800 (Thermo Fisher Scientific, Fremont, CA, U.S.A.), anti-human CD3 rabbit polyclonal antibody at 1:200 and anti-human HLA-DR alpha chain mouse monoclonal antibody (clone: TAL.1B5) at 1:500 (Dako Japan, Bunkyo-ku, Tokyo, Japan). We used the mesenteric lymph node of a normal beagle as a positive control before staining clinical samples and confirmed antibody cross-reactivity morphologically and anatomically. Tumor tissues were fixed in $10 \%$ neutral buffed formalin and embedded in paraffin in the routine manner. A series of $2-\mu \mathrm{m}$ sections of each sample was made and used for hematoxylin and eosin staining and immunohistochemical staining.

Immune cell counts were measured by one researcher in a blind fashion without information on the patients. The number of each immune cell type was counted at $\times 400$ magnification in 5 fields $[3,11]$. Because some studies reported that lymphocytes have different functions depending on the infiltrating area in tumor tissue and some of the collected tissues did not contain tumor margin, observed fields were randomly chosen from the intratumoral area rather than the peritumoral area [23]. CD3, CD20 and HLA-DR scores were defined as the mean number of positive cells in the fields and compared between tumor histological types.

The clinical outcomes of 51 malignant tumor patients were obtained from medical records and telephone interviews of owners or referring veterinarians. We determined death due to tumor to be the endpoint of this survival analysis. Patients who had postoperative chemotherapy $(n=3)$ and radiotherapy $(n=1)$ were not taken into consideration because of their influence on patient prognosis. Then, the relationship between $\mathrm{CD}^{+}$cell infiltration and death due to tumor was analyzed.

Yates $2 \times 2$ Chi square tests, Yates $m \times n$ Chi square tests and Fisher's exact tests were used to compare clinical features. Mann-Whitney U tests were used to compare age and body weight between groups and to clarify the difference in immune cell infiltration scores. Survival curves were generated by the Kaplan-Meier estimation method and compared by log-rank tests for overall survival. Variables that showed $P<0.1$ in univariate analysis were subjected to a Cox regres- sion procedure for multivariate analyses. A $P$ value $<0.05$ was considered statistically significance in all tests. These statistical analyses were done with ystat2008 and StatView-J 5.0.

The situations of the leukocyte infiltrations were apparently different between the malignant tumor and benign tumor groups. Significantly higher number of infiltrating $\mathrm{CD}^{+} \mathrm{T}$ cells were observed in the malignant tumor tissues $(P<0.05$; Fig. 1A). On the other hand, the number of infiltrating $\mathrm{CD} 20^{+}$cells showed no difference between the two groups (Fig. 1B). HLA-DR ${ }^{+}$cells in the tissues showed slightly higher infiltration in the malignant tumors than benign tumors; however, the difference was not statistically significant (Fig. 1C). According to these comparisons, hypothesizing that the increase in $\mathrm{T}$ cell infiltration had a potential relationship with tumor malignancy, we determined the cut-off value (26.48) for the CD3 score. This value was set from the score that contained $90 \%$ of benign tumor patients as a control host reaction against tumors, and patients with malignant mammary tumors were divided into groups, the high $\mathrm{CD}^{+}$infiltration and low $\mathrm{CD}^{+}$infiltration groups, and analyzed regarding clinical features and prognosis.

In the follow-up of patients, 17 dogs (including 18 malignant tumor tissues) were strongly suspected of dying from MGT. All these patients showed multiple recurrences or progression of distant metastasis with status worsening, although they were not completely confirmed by necropsy. Consequently, the median follow-up periods and numbers of deaths due to tumor, deaths from unknown causes, deaths from other causes, live dogs and dogs lost were as follows: 199 \pm 185 (range: 19-622) days for dogs that died of cancer $(n=18), 497 \pm 318$ (range: 4-924) days for dogs that died from unknown causes $(n=9), 1178 \pm 954$ (range: $226-2,798)$ days for dogs that died from other causes $(n=7), 1,003 \pm 249$ (range: 692-1,227) days for dogs that were still alive $(n=4)$ and $927 \pm 885$ (range: 16-2,743) days for dogs that lost during the follow-up study $(\mathrm{n}=13)$, respectively.

Among the analyzed factors, only high CD3 infiltration $(P<0.05)$ and metastasis $(P<0.001)$ showed a statistical difference in the multivariate analysis, although high CD3 infiltration was not considered as significant in the univari-
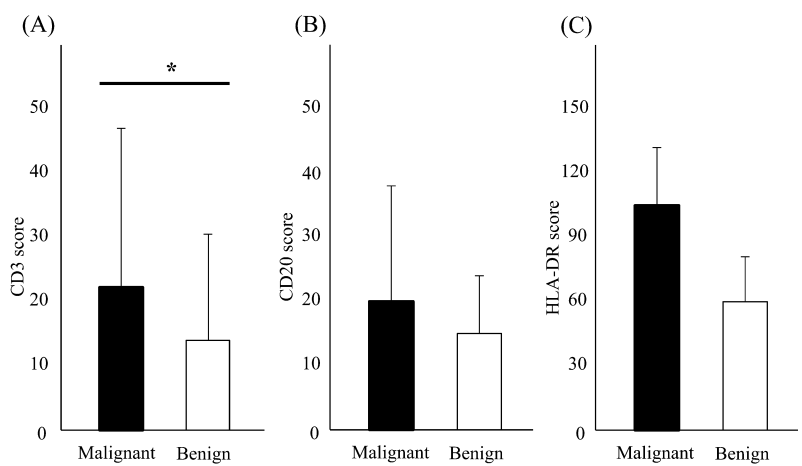

Fig. 1. The graphs show results of comparisons between the malignant tumor group and benign tumor group of CD3 (A), CD20 (B) and HLA-DR (C) scores. ${ }^{*} P<0.05$. 


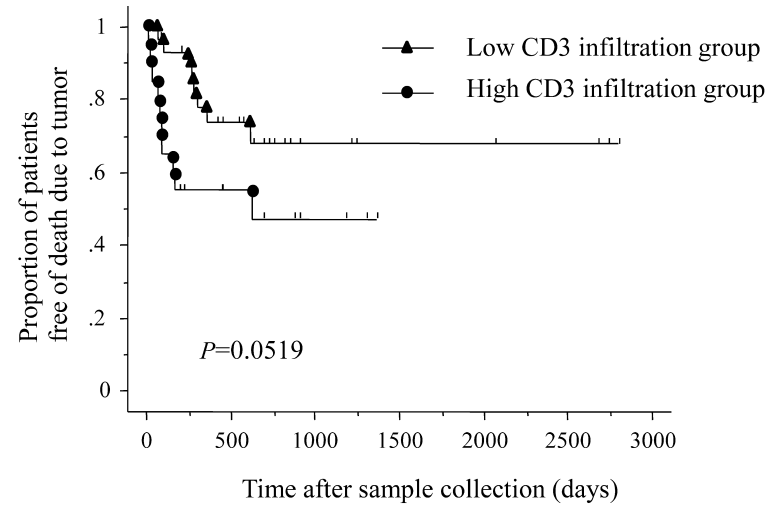

Fig. 2. Kaplan-Meier curves show a comparison of the proportions of patients free of death due to tumor after sample collection between the high CD3 infiltration and low CD3 infiltration groups. $P$ value represents the result from Log-rank test.

ate analysis. Using the Kaplan-Meier estimation method, $\mathrm{CD}^{+}$cell infiltration severity clearly separated patient prognosis (Fig. 2). Furthermore, the high CD3 infiltration group showed a significantly worse survival rate within 1 year, $19.6 \%$ (6/32), compared with that of the low CD3 infiltration group $(45 \%, 9 / 20)$.

The present study revealed the potential association of infiltrating $\mathrm{CD}^{+} \mathrm{T}$ lymphocytes with tumor malignancy in CMGTs. Studies of human solid tumors have reported both good and bad effects of T cell infiltration on tumor progression $[2,10,17,22,29,31]$. Focusing on the microenvironment of CMGTs, two reports showed the number of tumorinfiltrating leukocytes, both $\mathrm{T}$ cells and $\mathrm{B}$ cells, increased with malignant tumor progression $[8,16]$. In this study, we investigated the long-term prognosis of patients with malignant CMGT and confirmed the negative correlation of T cell infiltration and patient prognosis. With either analysis of surface marker or cytokine production, the above two reports also indicated that a type 2 immune reaction could related to the tumor malignancy. Secretions of cytokines, such as IL-4, 6, 10 and 13 from Th2 $\mathrm{CD}^{+}$cells and IL-10 and TGF- $\beta$ from regulatory $\mathrm{CD}^{+} \mathrm{T}$ cells, are considered to be potential contributors to tumor malignancy [10]. However, we could not elucidate whether increased malignancy of tumors caused a strong immune reaction or whether high infiltration of immune cells affected tumor malignancy from this study. Investigations of the change in tumor cell phenotype and infiltrating $\mathrm{T}$ cell population during malignant conversion will provide us more information to discuss mechanisms and functional roles of tumor-infiltrating $\mathrm{T}$ cells.

The numbers of B cells were reported to increase with malignant progression by some clinical investigations of human oncology $[5,34]$. However, in this study, the suggested difference related to tumor malignancy was not observed. Although the function of anti-tumor antibody produced by $\mathrm{B}$ cells is still controversial, many reports showed its relationship with tumor malignancy $[6,33]$.
Their cytokine and immunoglobulin production were considered to recruit innate immune cells, which make the surrounding environment more angiogenic and place it in a protumoral state, similar to areas of chronic inflammation $[6,9,30,33]$. Therefore, not only the genuine number of cells but also their activation status needs to be investigated to evaluate the role of infiltrating B cells.

The number of HLA-DR ${ }^{+}$antigen-presenting cells also showed no dynamic changes with tumor progression. The fact that the HLA-DR molecule is expressed on the cell membrane of some distinct cell populations including macrophages, dendritic cells and activated B lymphocytes should be mentioned here, which seemed to complicate the result. These cell populations have been reported to work distinctively in the tumor environment $[1,6,10,15,21,27]$. Therefore, further study of each population separately should be conducted to clarify the role of HLA-DR ${ }^{+}$cells as a next step.

In the comparison of clinicopathological features between the malignant and benign tumor patient groups, older dogs (mean age $11.3 \pm 2.6$ versus $9.7 \pm 2.1 ; P<0.001$ ) seemed to have a higher probability of malignancy. Moreover, the ratio of malignant cases in spayed dogs $(15 / 22)$ was significantly higher than that in intact dogs $(36 / 118$; $P<0.01)$. These tendencies were similar to those in previous reports $[12,20,28]$. In the follow-up study, 17 dogs died from cancer in the malignant tumor group (34\%), and this was also similar to the results of a previous study $(38.5 \%)$ [14]. It could be considered that the groups discussed here represented typical features of CMGTs free from biased sample collection. We also conducted a comparison of clinicopathological features between the high $\mathrm{CD}^{+}$infiltration group and low $\mathrm{CD}^{+}$infiltration group. However, we did not find any difference in clinicopathological features that potentially affect patient prognosis and tumor malignancy, such as age, ovarian status and clinical stage.

Tumor tissues consist of various types of cells, including tumor cells, inflammatory cells and stromal cells, and their active crosstalk has significant impact on the tumor behavior, as seen in normal organs. The surrounding environments of tumor tissues will change dramatically with their progression. In this study, $\mathrm{CD}^{+} \mathrm{T}$ cells accumulated with tumor progression, and their severe infiltration into tumor tissues correlated with poor prognosis of CMGT patients. The results in this study indicated the relationship of tumorinfiltrating $\mathrm{T}$ cells with CMGT malignancy. However, effects of other immune cells and the way how they influence tumor biology remain to be studied. Further investigations of the dynamic changes in the environment surrounding tumor cells will be required to expand our understandings of CMGT biology and to apply them to clinical practices.

\section{REFERENCES}

1. Allavena, P., Sica, A., Garlanda, C. and Mantovani, A. 2008. The Yin-Yang of tumor-associated macrophages in neoplastic progression and immune surveillance. Immunol. Rev. 222: 155-161. 
2. Al-Shibli, K., Al-Saad, S., Andersen, S., Donnem, T., Bremnes, R. M. and Busund, L. T. 2010. The prognostic value of intraepithelial and stromal CD3-, CD117- and CD138-positive cells in non-small cell lung carcinoma. Acta Pathol. Microbiol. Immunol. Scand. A 118: 371-382.

3. Amorim, R. L., Pinczowski, P., Neto, R. T. and Rahal, S. C. 2010. Immunohistochemical evaluation of prostaglandin E2 and vascular endothelial growth factor in canine cutaneous mast cell tumours. Vet. Comp. Oncol. 8: 23-27.

4. Balkwill, F. and Mantovani, A. 2001. Inflammation and cancer: back to Virchow? Lancet 357: 539-545.

5. Coronella-Wood, J. A. and Hersh, E. M. 2003. Naturally occurring B-cell responses to breast cancer. Cancer Immunol. Immunother. 52: 715-738.

6. DeNardo, D. G. and Coussens, L. M. 2007. Inflammation and breast cancer. Balancing immune response: crosstalk between adaptive and innate immune cells during breast cancer progression. Breast Cancer Res. 9: 212-221.

7. Dorn, C. R., Taylor, D. O., Schneider, R., Hibbard, H. H. and Klauber, M. R. 1968. Survey of animal neoplasms in Alameda and Contra Costa Counties, California. II. Cancer morbidity in dogs and cats from Alameda County.J. Natl. Cancer Inst. 40:307-318.

8. Estrela-Lima, A., Araújo, M. S., Costa-Neto, J. M., TeixeiraCarvalho, A., Barrouin-Melo, S. M., Cardoso, S. V., MartinsFilho, O. A., Serakides, R. and Cassali, G. D. 2010. Immunophenotypic features of tumor infiltrating lymphocytes from mammary carcinomas in female dogs associated with prognostic factors and survival rates. BMC cancer 10: 256-269.

9. Fernandez, M. F. 2005. Autoantibodies in breast cancer sera: candidate biomarkers and reporters of tumorigenesis. Cancer Lett. 230: 187-198.

10. Grivennikov, S. I., Greten, F. R. and Karin, M. 2010. Immunity, inflammation, and cancer. Cell 140: 883-899.

11. Han, L.Y., Fletcher, M.S., Urbauer, D.L., Mueller, P., Landen, C.N., Kamat, A.A., Lin, Y.G., Merritt, W.M., Spannuth, W.A., Deavers, M.T., De Geest, K., Gershenson, D.M., Lutgendorf, S.K., Ferrone, S. and Sood, A.K. 2008. HLA class I antigen processing machinery component expression and intratumoral T-Cell infiltrate as independent prognostic markers in ovarian carcinoma. Clin. Cancer Res. 14: 3372-3379.

12. Hellmen, E., Bergstrom, R., Holmberg, L., Spangberg, I. B., Hansson, K. and Lindgren, A. 1993. Prognostic factors in canine mammary tumors: a multivariate study of 202 consecutive cases. Vet. Pathol. 30: 20-27.

13. Horiuchi, Y., Tominaga, M., Ichikawa, M., Yamashita, M., Jikumaru, Y., Nariai, Y., Nakajima, Y., Kuwabara, M. and Yukawa, M. 2009. Increase of regulatory T cells in the peripheral blood of dogs with metastatic tumors. Microbiol. Immunol. 53: $468-474$.

14. Itoh, T., Uchida, K., Ishikawa, K., Kushima, K., Kushima, E., Tamada, H., Moritake, T., Nakao, H. and Shii, H. 2005. Clinicopathological survey of 101 canine mammary gland tumors: differences between small-breed dogs and others. J. Vet. Med. Sci. 67: 345-347.

15. Joyce, J. A. and Pollard, J. W. 2009. Microenvironmental regulation of metastasis. Nat. Rev. Cancer 9: 239-252.

16. Kim, J. H., Yu, C. H., Yhee, J. Y., Im, K. S. and Sur, J. H. 2010. Lymphocyte infiltration, expression of interleukin (IL)1, IL-6 and expression of mutated breast cancer susceptibility gene-1 correlate with malignancy of canine mammary tumours. J. Comp. Pathol. 142: 177-186.

17. Kobayashi, N., Hiraoka, N., Yamagami, W., Ojima, H., Kanai, Y., Kosuge, T., Nakajima, A. and Hirohashi, S. 2007. FOXP3 ${ }^{+}$ regulatory $\mathrm{T}$ cells affect the development and progression of hepatocarcinogenesis. Clin. Cancer Res. 13: 902-911.

18. Kumaraguruparan, R., Prathiba, D. and Nagini, S. 2006. Of humans and canines: Immunohistochemical analysis of PCNA, Bcl-2, p53, cytokeratin and ER in mammary tumours. Res. Vet. Sci. 81: 218-224.

19. Kumaraguruparan, R., Karunagaran, D., Balachandran, C., Manohar, B. M. and Nagini, S. 2006. Of humans and canines: a comparative evaluation of heat shock and apoptosis-associated proteins in mammary tumors. Clin. Chim. Acta 365: 168-176.

20. Lana, E. L., Rutteman, G. R. and Withrow, S. J. 2007. Tumors of mammary gland. pp. 619-636. In: Small Animal Clinical Oncology, 4th ed. (Withrow, S. J. and Vail D. M. eds.), Saunders, Philadelphia.

21. Mantovani, A., Marchesi, F., Porta, C., Sica, A. and Allavena, P. 2007. Inflammation and cancer: breast cancer as a prototype. Breast 16: S27-33.

22. Marrogi, A. J., Munshi, A., Merogi, A. J., Ohadike, Y., ElHabashi, A., Marrogi, O. L. and Freeman, S. M. 1997. Study of tumor infiltrating lymphocytes and transforming growth factorbeta as prognostic factors in breast carcinoma. Int. J. Cancer 74: 492-501.

23. Menegaz, R. A., Michelin, M. A., Etchebehere, R. M., Fernandes, P. C. and Murta, E. F. 2008. Peri- and intratumoral $\mathrm{T}$ and B lymphocytic infiltration in breast cancer. Eur. $J$. Gynaecol. Oncol. 29: 321-326.

24. Misdorp, W., Else, R. W., Hellmen, E. and Lipscomb, T. P. 1999. Histological Classification of Mammary Tumors of the Dog and Cat, Armed Forces Institute of Pathology, Washington D.C.

25. Moe, L. 2001. Population-based incidence of mammary tumours in some dog breeds. J. Reprod. Fertil. 57: 439-443.

26. Mukaratirwa, S., Chikafa, L., Dliwayo, R. and Moyo, N. 2006. Mast cells and angiogenesis in canine melanomas: malignancy and clinicopathological factors. Vet. Dermatol. 17: 141-146.

27. Murdoch, C., Muthana, M., Coffelt, S. B. and Lewis, C. E. 2008. The role of myeloid cells in the promotion of tumour angiogenesis. Nat. Rev. Cancer 8: 618-631.

28. Philibert, J. C., Snyder, P. W., Glickman, N., Glickman, L. T., Knapp, D. W. and Waters, D. J. 2003. Influence of host factors on survival in dogs with malignant mammary gland tumors. $J$. Vet. Intern. Med. 17: 102-106.

29. Ropponen, K. M., Eskelinen, M. J., Lipponen, P. K., Alhava, E. and Kosma, V. M. 1997. Prognostic value of tumour-infiltrating lymphocytes (TILs) in colorectal cancer.J. Pathol. 182:318-324.

30. Tan, E. M. and Shi, F. D. 2003. Relative paradigms between autoantibodies in lupus and autoantibodies in cancer. Clin. Exp. Immunol. 134: 169-177.

31. Tefany, F. J., Barnetson, R. S., Halliday, G. M., McCarthy, S. W. and McCarthy, W. H. 1992. Immunocytochemical Analysis of the cellular infiltrate in primary regressing and non-regressing malignant melanoma. J. Invest. Dermatol. 97: 197-202.

32. Uva, P., Aurisicchio, L., Watters, J., Loboda, A., Kulkarni, A., Castle, J., Palombo, F., Viti, V., Mesiti, G., Zappulli, V., Marconato, L., Abramo, F., Ciliberto, G., Lahm, A., Monica, N. L. and Rinaldis, E. D. 2009. Comparative expression pathway analysis of human and canine mammary tumors. BMC Genomics 10: $135-154$.

33. Visser, K. D., Eichten, A. and Coussens, L. M. 2006. Paradoxical roles of the immune system during cancer development. Nat. Rev. Cancer 6: 24-37.

34. Wong, P. Y., Staren, E. D., Tereshkova, N. and Braun, D. P. 1998. Functional analysis of tumor-infiltrating leukocytes in breast cancer patients. J. Surg. Res. 76: 95-103. 